\title{
The future of heavy ions at the high energy frontier - ALICE upgrades and more
}

\section{Thomas Peitzmann*}

Utrecht University/Nikhef, Utrecht, The Netherlands

E-mail: t.peitzmann@uu.nl

I discuss the future of heavy-ion physics at high energy. I briefly mention some aspects of the current status of the field and then concentrate on the role of detector upgrades at the LHC. I summarise the relevance of upgrades of the ATLAS and CMS experiments for heavy ions, and then discuss in detail the upgrade programme of ALICE, which is dedicated to heavy-ion physics. Finally I sketch the upcoming opportunities at a new collider at still higher energy.

7th International Conference on Physics and Astrophysics of Quark Gluon Plasma

1-5 February, 2015

Kolkata, India

${ }^{*}$ Speaker. 


\section{Introduction}

Since the startup of LHC a new chapter of heavy-ion physics at the highest energy has opened, which has formed a substantial part of the overall LHC physics program. ALICE, the dedicated heavy-ion experiment, has carried through its rich measurement program, but also the two big pp experiments, CMS and ATLAS, have established themselves as important players in heavy-ion physics. Together, these experiments have collected and published a vast number of results, which have significantly furthered our understanding of strongly interacting matter at high density and temperature.

The purpose of this paper is not to provide a review, but it is useful to remind ourselves of some of the interesting features of the results obtained so far. The bulk matter produced in central $\mathrm{Pb}-\mathrm{Pb}$ collisions at the LHC qualitatively shares many characteristics with that produced at lower energy at RHIC. Transverse momentum spectra of identified hadrons show the signs of collective radial expansion [1]. In general, the collisions seem to be more violent, with larger multiplicity density, higher average transverse momentum of produced particles and stronger collective motion. The relative abundances of hadron species [2] can be described by statistical hadronization models with just a single "temperature" parameter with a value close to the estimates of the critical temperature, in fact very similar to the one obtained at RHIC. If one looks at the details, there are still a number of "minor" puzzles, like in this latter case the fact that the proton yields do not seem to match the same model predictions, that describe the other species. Measurements of azimuthal anisotropies, which have lead to the postulation of the near-perfect liquid behaviour of matter produced at RHIC, have also revealed strong elliptic flow at the LHC [3]. The elliptic flow parameter $v_{2}$ as a function of $p_{T}$ seems to have a very similar behaviour as at RHIC, which together with the stronger radial flow leads to a larger magnitude of the integrated $v_{2}$.

In the realm of hard probes, the measurements open up new possibilities due to the higher beam energy and the consequently higher yield, but the exploitation of these has just begun. Again a suppression of $J / \psi$ production in nuclear collisions has been observed [4], however, quantitatively the suppression appears to be weaker than at RHIC. The most likely explanation for this behaviour is that, while the suppression of charmonium from nuclear absorption and additional Debye-screening in the quark-gluon plasma was the dominant feature at RHIC energies and below, at the LHC this is at least partially compensated by a new production mechanism, the recombination of independently produced charm quarks [5].

Measurements of jet quenching have also been performed, e.g. via the nuclear modification of single hadron yields [6] and also via the study of the asymmetry of dijets [7]. In the $p_{T}$-region of $5-7 \mathrm{GeV} / c$ single hadrons are even more strongly suppressed than at RHIC. In this region of maximum suppression, hadron production is likely dominated by emission from the surface, as the bulk medium appears to be extremely opaque. Even more interestingly, the nuclear modification factor $R_{A A}$ shows a monotonous increase towards still higher $p_{T}$, unlike at RHIC. This may mainly reflect the fact that the underlying parton spectra are flatter at LHC, and this feature should allow to put more stringent requirements on theoretical model descriptions than was possible with the featureless $R_{A A}$ from RHIC. However, the theoretical description of these data by parton energy loss models is still an open question.

Yet more information should come from the study of heavy-flavour hadrons. Models predict 
a quark mass dependence of energy loss, and so charm (and even more beauty) hadrons should allow further testing of the models. They should in fact be more penetrating and could thus probe more of the true medium density, as they might not suffer so much from a surface bias as light hadrons. First results of the nuclear modification factor of charmed mesons have been obtained [8], which show that also D mesons are strongly suppressed, in fact similarly to light hadrons. At intermediate $p_{T}$, hints of a different behaviour is seen, that can be associated with the larger charm quark mass. Also for studies of elliptic flow, charm mesons are very sensitive probes. Due to their larger mass and correspondingly smaller cross section, it is expected that they should not reach equilibrium with the bulk system as easily. From first measurements of ALICE [9], also the D mesons show a significant elliptic flow strength, not very different in magnitude from that of light hadrons. As examples of the advanced measurements at LHC, these results of charm measurements are shown in Fig. 1. To use these and similar results to quantitatively constrain models and by that the properties of the hot and dense matter further will require higher statistics but also upgraded detectors to allow a reduction of the systematic uncertainties.
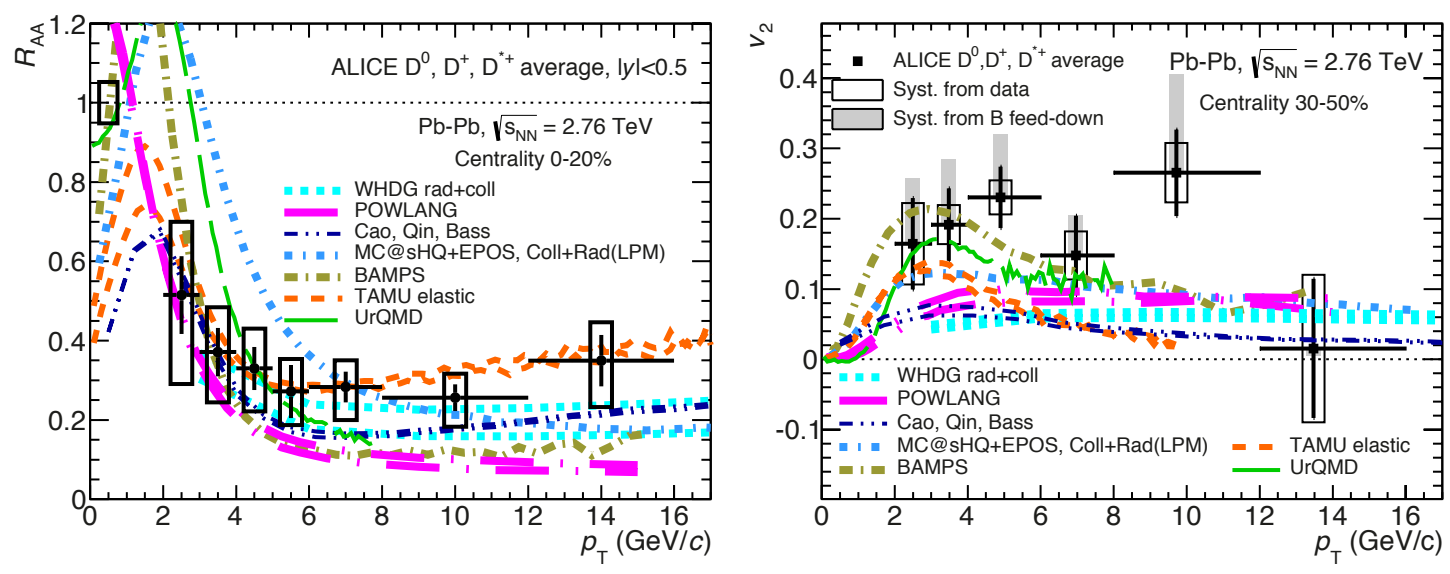

Figure 1: Charm mesons measurements compared to various model predictions (from [9]). Left: Nuclear modification factor $R_{A A}$ of $\mathrm{D}$ mesons as a function of $p_{T}$ in central $\mathrm{Pb}-\mathrm{Pb}$ collisions. Right: Elliptic flow strength $v_{2}$ of $\mathrm{D}$ mesons as a function of $p_{T}$.

\section{Upgrades for heavy ions at LHC}

\subsection{CMS and ATLAS upgrades}

Both CMS and ATLAS have no plans for upgrades of their detectors which are dedicated to heavy-ion measurements. Still, these experiments have very substantial upgrade programs [10, 11], which are specifically geared towards measurements in pp with high luminosity. Heavy-ion measurements will then certainly also profit from the improved detectors. In particular, all measures taken in these experiments to deal with multi-event pile up, which will be a major challenge in the high-luminosity era of LHC, will also improve the performance in the environment of heavy-ion collisions with their much higher multiplicity. Many hardware upgrades at the LHC can only be performed in longer shutdown periods. One of these (long shutdown 1) has just happened recently, 
while two other (long shutdown 2 and 3 ) are scheduled for $\approx 2019$ and $\approx 2024$, respectively. I will denote these periods below with their abbreviations, e.g. LS 3.

The CMS experiment plans to implement 4 new layers of silicon pixel detectors in 2016/17 and will upgrade the full silicon tracker in LS 3. Regarding the calorimeters, the granularity for triggering will be improved in 2015, and a new endcap and an electronics upgrade will be installed in LS 2. Again in 2015, the acceptance of the muon detectors will be enlarged and the trigger improved. An additional increase in acceptance will be performed in LS3. Furthermore, the trigger will be upgraded in 2015 to be able to handle the higher $\mathrm{Pb}-\mathrm{Pb}$ luminosity, and in LS3 more selective triggers will be implemented, as e.g. a level 1 track trigger, which should also be very useful in heavy-ion collisions.

The ATLAS collaboration upgrades their tracking in three steps: An additional first pixel layer has already been installed in LS 1, while a fast tracking trigger will be implemented in LS 2. Finally, in LS3 ATLAS will install a completely new tracker based entirely on silicon, which should lead to a reduced material budget and possibly a larger acceptance. In LS2 both the calorimeter and the muon system will be upgraded to have higher segmentation for the trigger, and the muon measurements will be further improved by installing a new small wheel for forward muons. Furthermore, the trigger system will be upgraded with new processors, which should allow trigger decisions taking into account the full event information.

\subsection{ALICE upgrades - the baseline}

The situation is very different for ALICE, which is a dedicated heavy-ion experiment. Naturally, the planned upgrades are all directly motivated by a specific physics program in heavy-ion collisions. Overall, an upgraded ALICE setup at high luminosity should allow

1. precision studies of primary charm (open and hidden) including flow and correlations,

2. measurements of low mass lepton pairs and thermal photons,

3. studies of jet modification via gamma-jet and jet-jet correlations including particle ID, and

4. the search for heavy nuclear states.

ALICE is preparing an upgrade program that builds on the existing strengths of the experimental setup, such as excellent tracking performance, in particular at low momenta, efficient secondary vertex reconstruction, very low material budget, and excellent particle identification. The upgraded ALICE detector will significantly improve the performance in most of these areas, except for particle identification, which will be preserved. Furthermore, the upgraded experimental setup will have enhanced rate capabilities to allow an inspection of $\mathrm{Pb}-\mathrm{Pb}$ collisions at an interaction rate of $50 \mathrm{kHz}$. This is necessary to be able to exploit the physics potential, because many of the signals have an irreducibly low signal-to-background ratio requiring a high statistics measurement, but at the same time making them untriggerable. Fortunately, the signal-to-background for these observables will be much better in pp, such that only a moderately higher luminosity $(\approx 200 \mathrm{kHz}$ ) will be needed for reference measurements. At appropriate luminosity the upgraded apparatus will allow us to collect sufficient reference statistics in short reference runs. Crucial parts of the upgrade are the development and implementation of a significantly improved inner tracking system (ITS), the 
Table 1: Summary of the physics reach: minimum accessible $p_{\mathrm{T}}$ and relative statistical uncertainty in $\mathrm{Pb}-\mathrm{Pb}$ collisions for an integrated luminosity of $\left(10 \mathrm{nb}^{-1}\right.$. For heavy flavour, the statistical uncertainties are given at $p_{\mathrm{T}}=2 \mathrm{GeV} / c$. For elliptic flow measurements, the value of $v_{2}$ used to calculate the relative statistical uncertainty $\sigma_{v_{2}} / v_{2}$ is given in parenthesis.

\begin{tabular}{|c|c|c|}
\hline Observable & $p_{\mathrm{T}}^{\min }(\mathrm{GeV} / c)$ & statistical uncertainty \\
\hline & leavy Flavour & (at $2 \mathrm{GeV} / c)$ \\
\hline D meson $R_{\mathrm{AA}}$ & 0 & $0.3 \%$ \\
\hline $\mathrm{D}_{\mathrm{s}}$ meson $R_{\mathrm{AA}}$ & $<2$ & $3 \%$ \\
\hline D meson from B decays $R_{\mathrm{AA}}$ & 2 & $2 \%$ \\
\hline $\mathrm{J} / \psi$ from B $R_{\mathrm{AA}}$ & 1 & $5 \%$ \\
\hline $\mathrm{B}^{+}$yield & 3 & $10 \%(>3 \mathrm{GeV} / c)$ \\
\hline$\Lambda_{\mathrm{c}} R_{\mathrm{AA}}$ & 2 & $15 \%$ \\
\hline Charm baryon-to-meson ratio & 2 & $15 \%$ \\
\hline$\Lambda_{\mathrm{b}}$ yield & 7 & $20 \%(7-10 \mathrm{GeV} / c)$ \\
\hline D meson elliptic flow $\left(v_{2}=0.2\right)$ & 0 & $3 \%$ \\
\hline $\mathrm{D}_{\mathrm{S}}$ meson elliptic flow $\left(v_{2}=0.2\right)$ & $<2$ & $8 \%$ \\
\hline D from B elliptic flow $\left(v_{2}=0.1\right)$ & 2 & $20 \%$ \\
\hline $\mathrm{J} / \psi$ from B elliptic flow $\left(v_{2}=0.1\right)$ & 1 & $30 \%$ \\
\hline$\Lambda_{\mathrm{c}}$ elliptic flow $\left(v_{2}=0.15\right)$ & 3 & $20 \%(3-6 \mathrm{GeV} / c)$ \\
\hline \multicolumn{3}{|c|}{ Dielectrons } \\
\hline Temperature (intermediate mass) & & $10 \%$ \\
\hline Elliptic flow $\left(v_{2}=0.1\right)$ & & $10 \%$ \\
\hline Low-mass spectral function & 0.3 & $20 \%$ \\
\hline \multicolumn{3}{|c|}{ Hypernuclei } \\
\hline${ }_{\Lambda}^{3} \mathrm{H}$ yield & 2 & $2 \%$ \\
\hline
\end{tabular}

replacement of the TPC readout chambers with GEMs, a general upgrade of the trigger detectors (e.g. V0, T0) and of all readout electronics, and a new system integrating DAQ, High-Level Trigger and offline computing. Examples for the expected performance for important observables are given in Table 1. More information on the improvements and further details on the ALICE upgrade can be found in [12].

Another new detector, the Muon Forward Tracker, will be implemented to further enhance the measurement capabilities for the signals mentioned above via the improved forward measurement of quarkonia and low-mass dimuons, as well as heavy-flavour muons. Installation of these detector upgrades is foreseen for the LHC long shutdown 2, implying a measurement program for $\mathrm{pp}, \mathrm{p}-\mathrm{Pb}$, and $\mathrm{Pb}-\mathrm{Pb}$ extending up to at least $\approx 2026$ - a possible scenario for the measurement program is summarised in Table 2.

The existing Time Projection Chamber (TPC) [13] is a unique element of the current ALICE apparatus with its continuous tracking and particle identification. However, with its long drift time it is limiting the rate capabilities. Using the multi-wire readout chambers, a gated operation (at a maximum of $3.5 \mathrm{kHz}$ ) is necessary to limit ion feedback to the drift volume, which would otherwise lead to intolerable space-charge distortions. To be able to inspect a significantly higher 
Table 2: Possible running scenario for the operation of the upgraded ALICE detector.

\begin{tabular}{llll}
\hline year & beams & int. luminosity & remarks \\
\hline 2019 & $\mathrm{~Pb}-\mathrm{Pb}$ & $2.85 \mathrm{nb}^{-1}$ & \\
2020 & $\mathrm{~Pb}-\mathrm{Pb}$ & $2.85 \mathrm{nb}^{-1}$ & low magnetic field \\
2021 & $\mathrm{pp}$ & t.b.c. & reference run \\
2022 & - & - & long shutdown 3 \\
2023 & - & - & long shutdown 3 \\
2024 & $\mathrm{~Pb}-\mathrm{Pb}$ & $2.85 \mathrm{nb}^{-1}$ & \\
2025 & $\mathrm{~Pb}-\mathrm{Pb}$ & $1.42 \mathrm{nb}^{-1}$ & $50 \%$ of beamtime \\
& $\mathrm{p}-\mathrm{Pb}$ & $50 \mathrm{nb}^{-1}$ & $50 \%$ of beamtime \\
2026 & $\mathrm{~Pb}-\mathrm{Pb}$ & $2.85 \mathrm{nb}^{-1}$ & \\
\hline
\end{tabular}

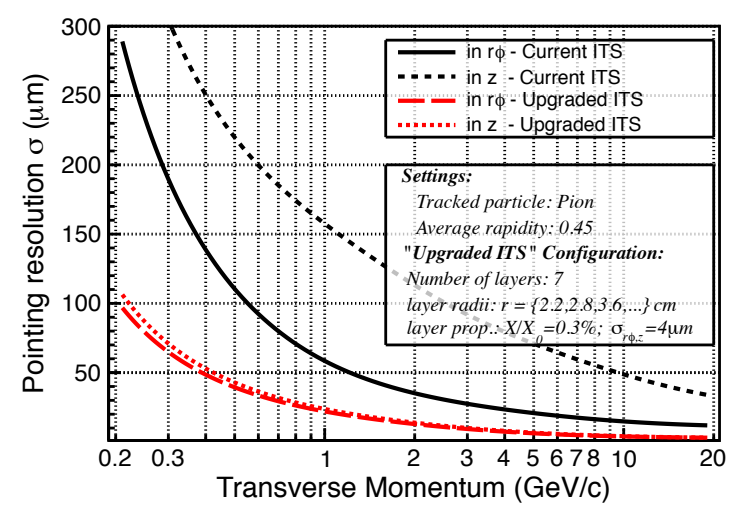

Figure 2: Track impact parameter resolution in $r \phi$ and $z$ for the current (black) and upgraded (red) ITS.

interaction rate, new readout chambers based on triple-GEMs will be developed. These would be continuously read out (no gating) and should have small enough ion feedback to allow an operation at $50 \mathrm{kHz}$ interaction rate. R\&D on the GEM readout chambers is ongoing with an emphasis on achieving the projected low ion feedback and in particular on demonstrating the necessary stability in a high multiplicity/high rate environment. In a pipelined readout data would be shipped from the TPC front-end at $10 \mathrm{MHz}$ for later inspection in a high level trigger system (HLT). The readout electronics of all existing detector systems will be modified to accommodate the rate capabilities. Some systems will likely share the pipelined electronics with the TPC, other detectors, e.g. the electromagnetic calorimeter (EMCal), might preferably be readout on L1 triggers provided by a hardware trigger unit.

The high rate upgrade scenario results in $\approx 1 \mathrm{~TB} / \mathrm{s}$ of input to the online systems, where most of the data originates from the TPC readout. This necessarily will be reduced in particular for the TPC information via cluster finding and cluster-track association, as well as standard Huffman encoding. An average output rate to tape storage of the order of $10 \mathrm{~GB} / \mathrm{s}$ after this data compression should be achievable.

A completely new Inner Tracking System (ITS) will be developed. The most important features of the new design in addition to enhanced rate capabilities will be: 


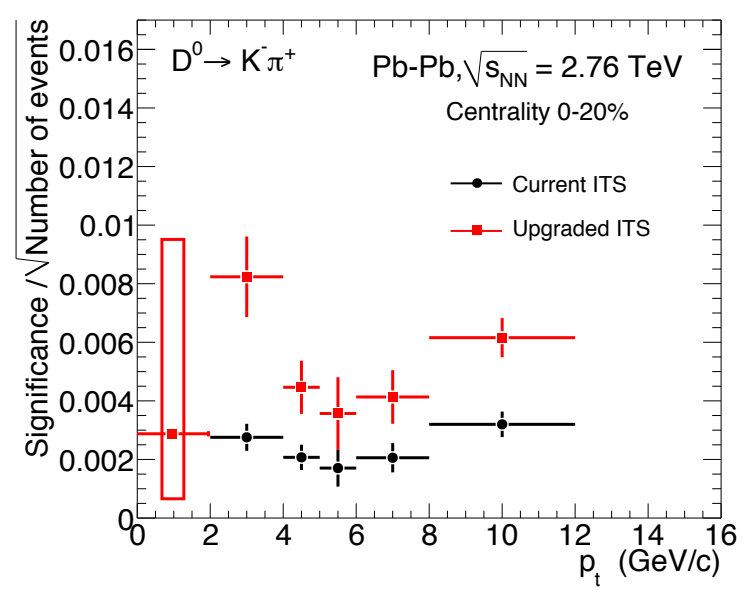

Figure 3: Significance for the measurement of the $D^{0}$ as a function of $p_{T}$ normalised to the square root of the number of events. The performance of the upgraded ITS is compared to that of the current detector.

- A decrease of the radial distance of the first layer to the beam to $22 \mathrm{~mm}$ (from $39 \mathrm{~mm}$ ),

- a reduction of the layer thickness possibly down to $50 \mu \mathrm{m}$ (from $350 \mu \mathrm{m}$ ), and

- a higher granularity with pixel sizes as small as $20 \times 20 \mu \mathrm{m}^{2}$.

This should be achievable by implementing seven layers of Monolithic Active Pixel Sensors and further optimisation of cabling, mechanical and cooling structures.

The new ITS will improve the secondary vertex resolution by a factor of $\approx 3$ (see Fig. 2) and allow measurements down to much lower values of $p_{T}$. Furthermore the high efficiency and low contamination of the new ITS should allow for enhanced level 2 trigger capabilities. It should give access to the measurement of charmed and beauty baryons and to the discrimination of prompt and secondary heavy quark production.

Fig. 3 shows a comparison of the expected significance for a $D^{0}$ measurement with the upgraded ITS to that expected with the current detector - in particular at low $p_{T}$ the performance improves significantly. Even more crucial is the upgrade for a measurement of the $\Lambda_{c}$ baryon in heavy-ion collisions, which cannot be measured with the current apparatus. In Fig. 4 one can see the expected statistical uncertainty for a measurement of the $\Lambda_{c} / D^{0}$ ratio in central $\mathrm{Pb}-\mathrm{Pb}$ collisions with the upgrade.

As another important example of the estimated performance Fig. 5 shows simulations of an elliptic flow measurement of prompt and secondary D mesons for $10 \mathrm{nb}^{-1}$ (left) and for $0.1 \mathrm{nb}^{-1}$ (right), the former of which would only be accessible with the upgrade.

The improved background rejection and the better identification of heavy-flavour electrons would also enable us to perform unprecedented measurements of low-mass dileptons. Fig. 6 shows mass spectra of the excess dielectrons after the subtraction of the combinatorial background and the correlated background from hadron decays (including heavy flavour). The errors on the contribution of these background sources dominates the measurement without the upgrade (left panel). These systematic errors would be significantly reduced with the upgraded ITS (right panel). In addition, the comparison of the two panels demonstrates the improvement in statistical errors from 


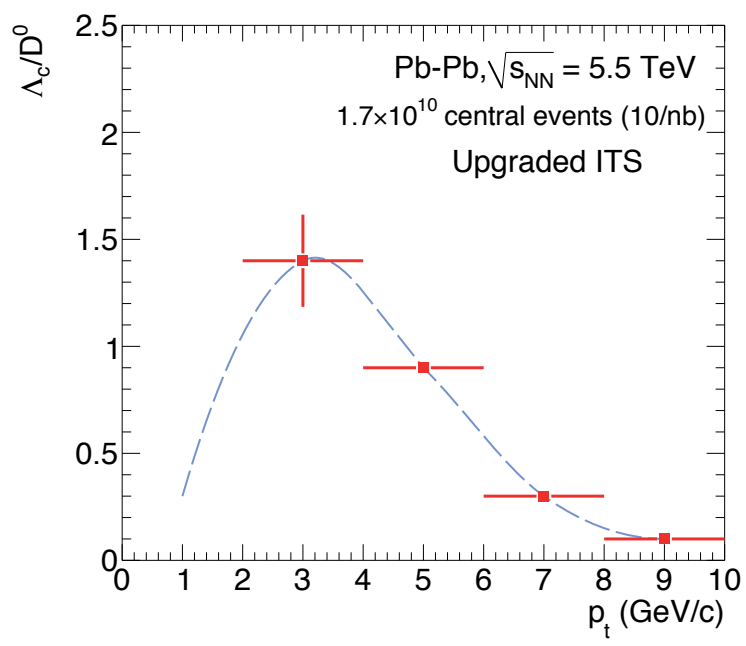

Figure 4: Possible measurement with the upgrade of the $\Lambda_{c} / D^{0}$ ratio as a function of $p_{T}$ for central $\mathrm{Pb}-\mathrm{Pb}$ collisions. The points are drawn on a line that captures the behaviour of the measured $\Lambda / K_{S}^{0}$ ratio.
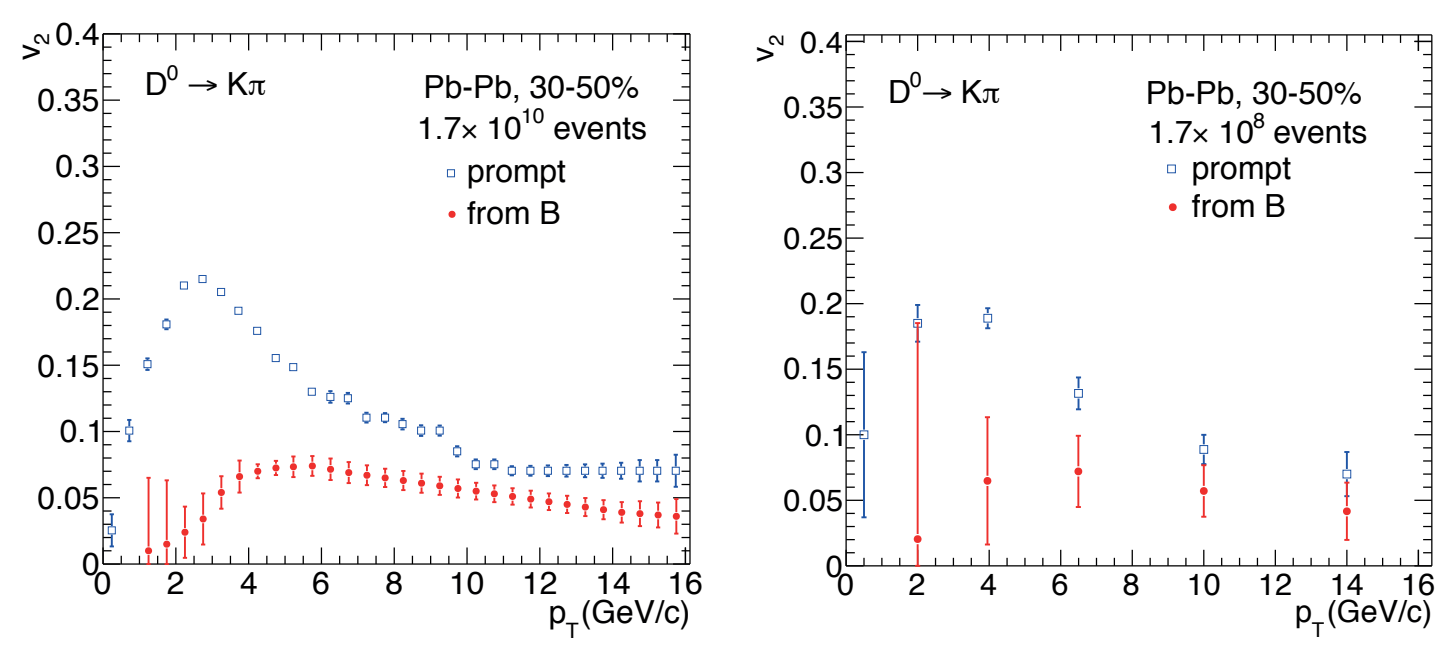

Figure 5: Estimated statistical uncertainties on $v_{2}$ of prompt and secondary $\mathrm{D}^{0}$ mesons for $1.7 \cdot 10^{10}$ events (left) in the $30-50 \%$ centrality class, which correspond to $10 \mathrm{nb}^{-1}$, and for $1.7 \cdot 10^{8}$ events (right), which correspond to about $0.1 \mathrm{nb}^{-1}$.

the enhanced rate capabilities. These measurements would profit further from a dedicated run at lower magnetic field to improve the detector acceptance at low mass and low $p_{T}$.

More details on the design and the physics program and performance of the new ITS is described in [14].

The installation of the Muon Forward Tracker (MFT), a tracking system complementing the Muon Spectrometer, will improve the physics performance of the muon measurements at forward rapidities $(-4.0<\eta<-2.5)$. The MFT will be located upstream of the hadron absorber of the Muon Spectrometer, close to the new ITS, and will consist of five tracking planes built on the same monolithic active pixel technology as the new ITS. By adding tracking in front of the muon ab- 

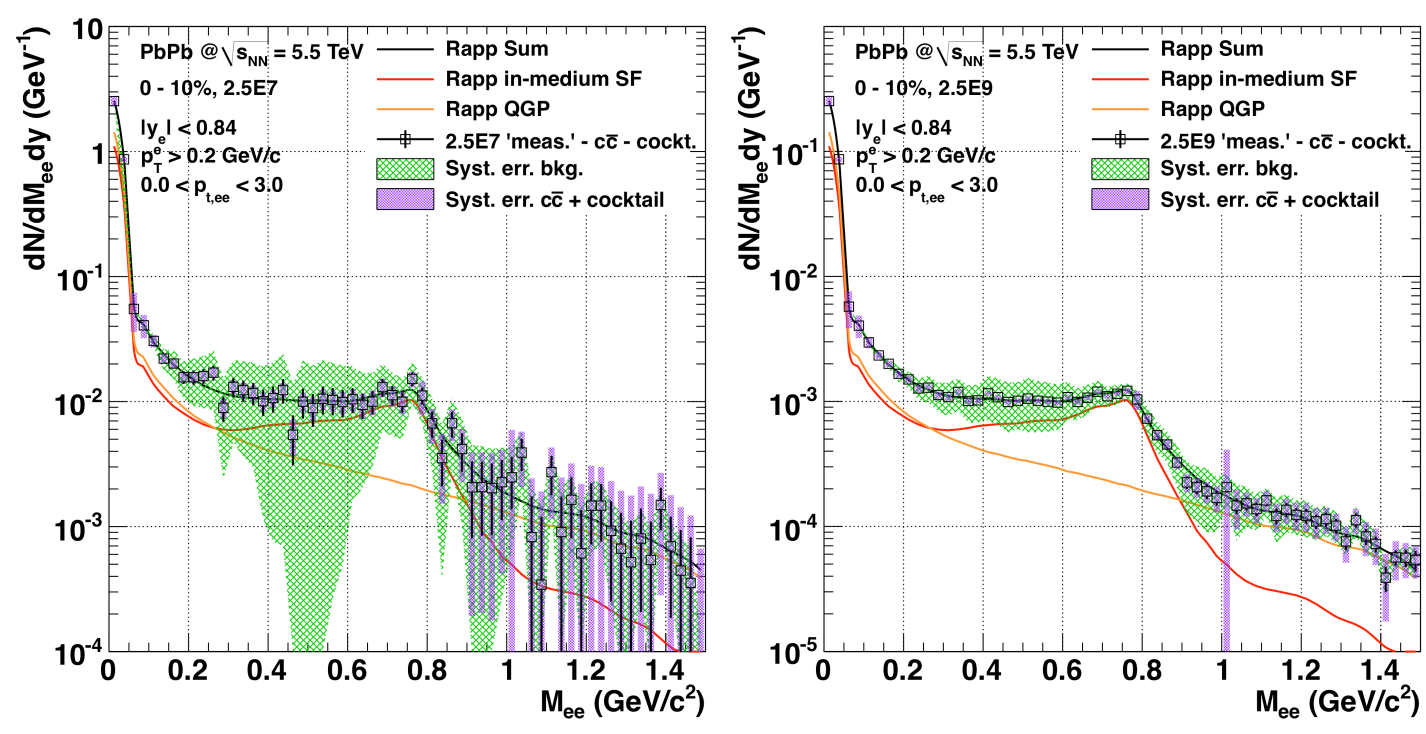

Figure 6: Invariant mass spectrum of excess $e^{+} e^{-}$pairs after the subtraction of known background sources for $0-10 \%$ most central $\mathrm{Pb}-\mathrm{Pb}$ collisions at $\sqrt{s_{N N}}=5.5 \mathrm{TeV}$. The green boxes show the systematic uncertainties from the combinatorial background subtraction, the magenta boxes indicate systematic errors related to the subtraction of the cocktail and charm contribution. The left panel shows estimates using the performance of the current ITS, the right panel the results for the upgraded ITS.
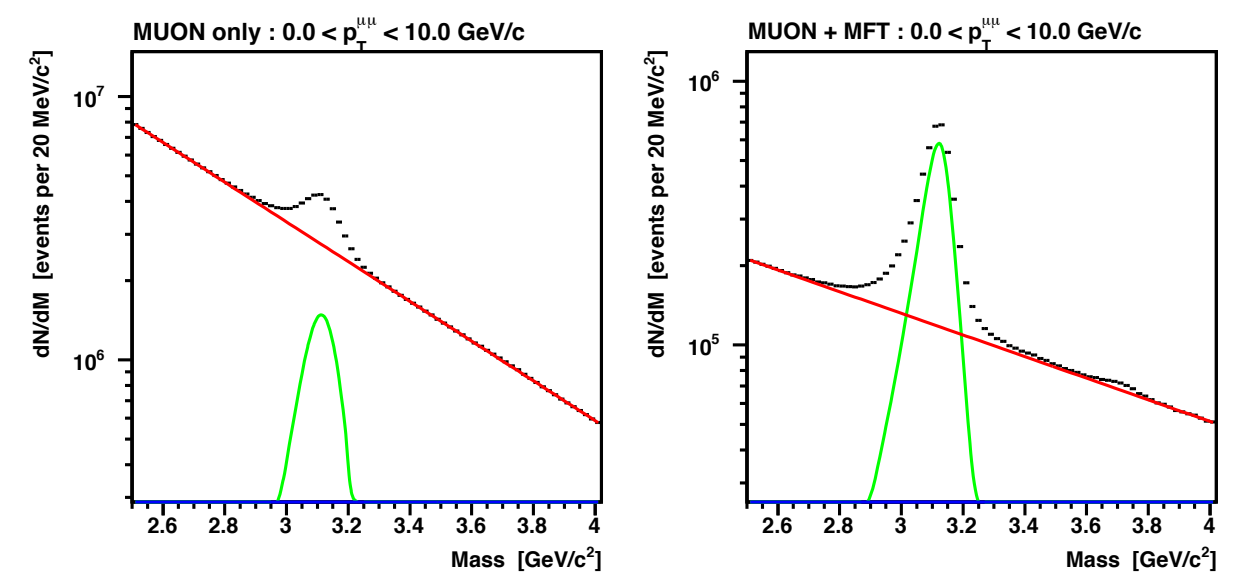

Figure 7: Mass distributions of dimuons in the vicinity of the $J / \psi$ in central $\mathrm{Pb}-\mathrm{Pb}$ collisions without MFT (left panel) and with MFT (right panel).

sorber, it would allow us to reconstruct possible secondary vertices of muon tracks. This would remove the largest limitation of the current forward muon measurements, enabling the identification of $J / \psi$ from $B$ decays, and would improve the resolution and signal-to-background ratio resulting in better general performance for measurements of quarkonia and low mass dileptons. The improvement in the signal-to-background ratio for charmonium states is demonstrated in Fig. 7. The left panel shows the performance without the MFT - here in particular the $\psi^{\prime}$ is not at all apparent. With the MFT (right panel), the background can be reduced dramatically, causing in particular 

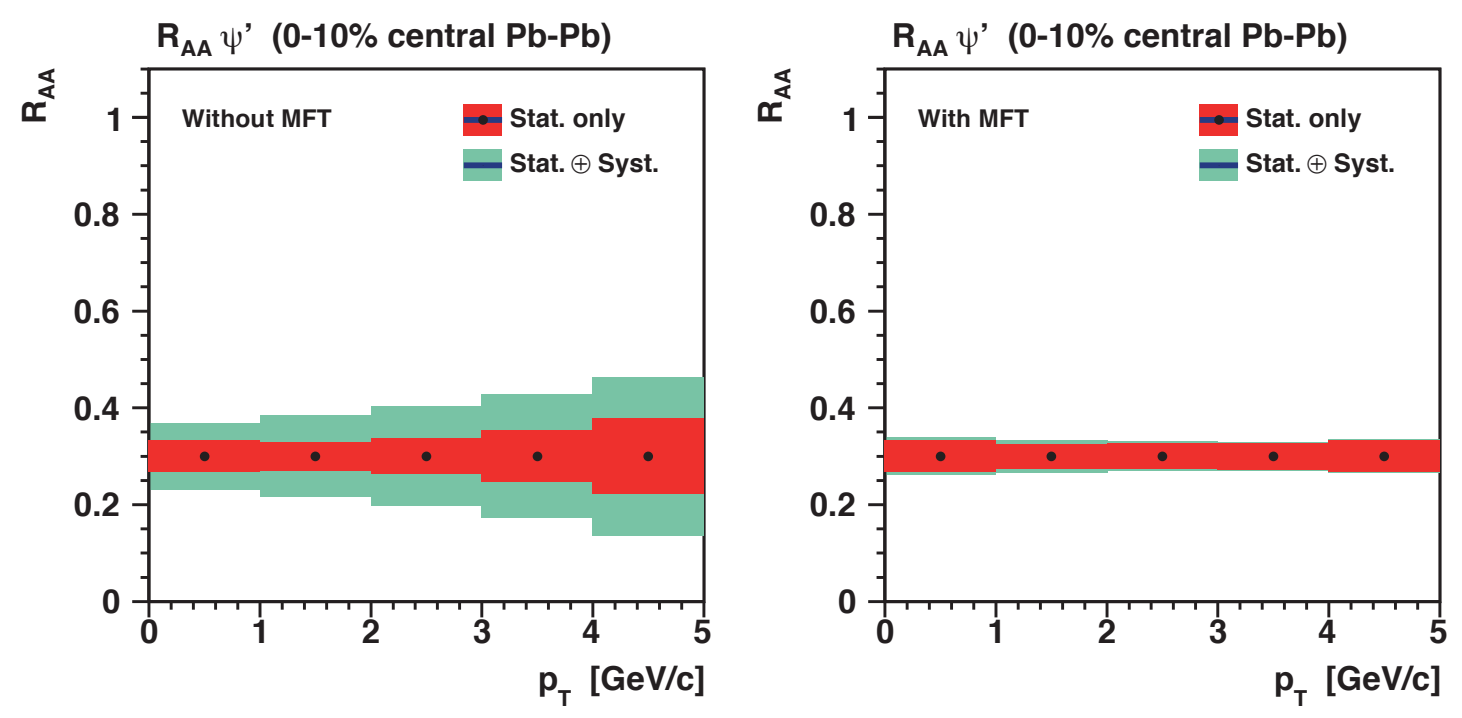

Figure 8: Expected statistical and systematical uncertainties of the nuclear modification factor $R_{A A}$ of the $\psi^{\prime}$ in central $\mathrm{Pb}-\mathrm{Pb}$ collisions without MFT (left panel) and with MFT (right panel).

the $\psi^{\prime}$ to be observable. More quantitatively, the signal-to-background ratio and systematic errors for the measurement of the $\psi^{\prime}$ in central $\mathrm{Pb}-\mathrm{Pb}$ collisions should improve by a factor of $3-10$ depending on the transverse momentum. The expected improvement for the measurement of the corresponding nuclear modification factor with the MFT compared to the performance of the existing muon arm alone is illustrated in Fig. 8. Details on the MFT performance can be found in [15].

\subsection{Forward direct photons}

For measurements at collider energies, the parton distributions are not very well constrained at low Bjorken $x$, and even more so for nuclei. DGLAP evolution predicts a dramatic increase of the gluon density, which would lead to fundamental problems, e.g. with unitarity. It is expected that non-linear effects (gluon fusion or recombination) would limit the growth and would lead to gluon saturation [16].

Gluon saturation should effect the total multiplicity of produced particles in high-energy collisions. Furthermore, the predicted scaling properties of the momentum scales should lead to a behaviour called geometric scaling. Both the multiplicities and the scaling properties of particle distributions have been measured and compared to saturation models. The results appear to be consistent with expectations from the models, but unfortunately these observables are not specific enough to provide a proof for gluon saturation.

More discriminatory power may come from more detailed studies of transverse momentum distributions and from two-particle angular correlations. The main interest lies here in the comparison of particle production in $\mathrm{pp}$ and $\mathrm{p}-\mathrm{A}$ collisions, as saturation effects should be stronger for the higher gluon density in nuclei. Specifically, one expects that 
- the nuclear modification factor in $\mathrm{p}-\mathrm{A}$ collisions should show a suppression of particle production $R_{p A}<1$ in a characteristic $p_{T}$ range, and

- the jet-like peak at $\Delta \phi=0$ usually observed in two-particle correlations in pp collisions should be modified (weakened and/or broadened) in nuclear collisions.

The interesting kinematic range is defined by the values of Bjorken $x$ and the corresponding saturation scale $Q_{s}$. The relevant production processes will be affected by saturation when $Q<Q_{s}$, which calls for small to intermediate momentum probes. However, as one would like to use a calculable probe as a reference, this excludes too low momentum transfers - ideally one would want to study momentum ranges, where perturbative QCD should be applicable. To access small $x$, which for leading-order processes on the parton level can be approximated as $x \approx 2 p_{T} \exp (-y) / \sqrt{s}$, particle production at large (i.e. forward) rapidities should be studied.

Transverse momentum spectra and angular correlations for neutral pions at forward rapidity have been studied in pp and d-A collisions at RHIC, and a suppression has been observed in the nuclear modification factor [17] and a suppression and broadening in the angular correlation [18]. However, the transverse momenta studied are still very small (on the order of $1 \mathrm{GeV}$ or only slightly higher), a momentum range where particle production is anyhow not well understood. In addition, the relation between the kinematic variables in the final state are only weakly correlated to the parton kinematic parameters due to fragmentation and possibly other final state modifications of particle production.

Similar studies at LHC should allow to study both higher $Q^{2}$ and smaller $x$ contributions, thus should be able to use well-defined particle production processes, while still being sensitive to saturation as the saturation scale should be much larger at the lower $x$ values. First measurements of hadron production at forward rapidities have been performed at the LHC, but results are not conclusive. The production of $\phi$ mesons shows a suppression in $\mathrm{p}+\mathrm{A}$ collisions compared to $\mathrm{pp}$
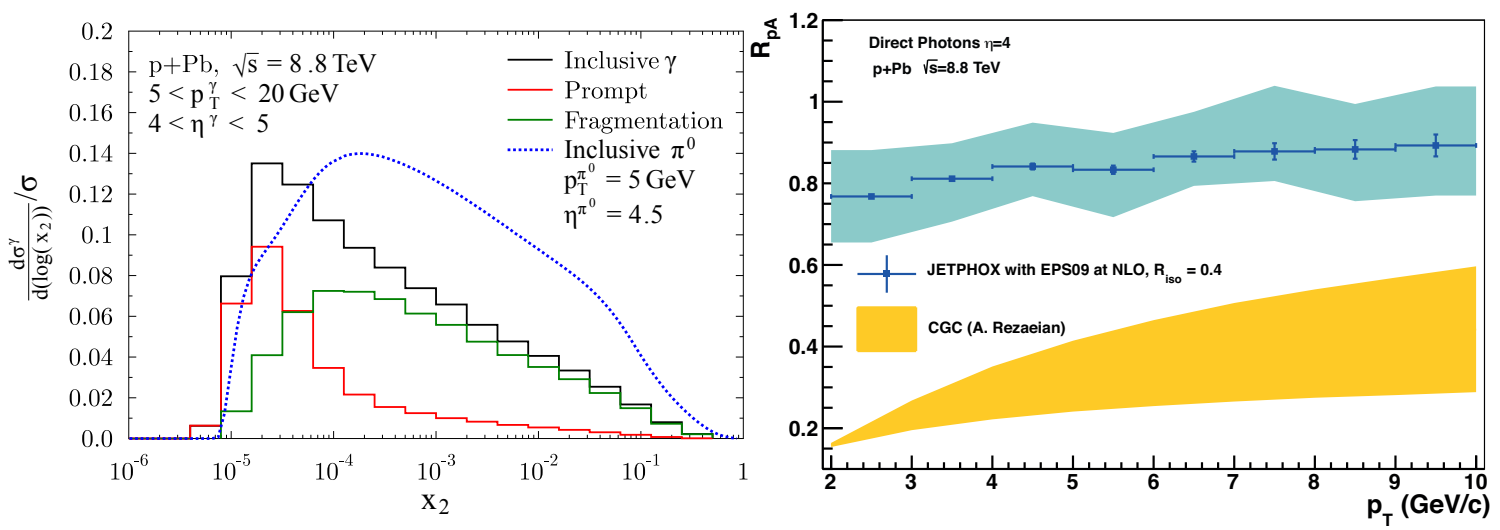

Figure 9: left: Distribution of $x_{2}$ (momentum fraction of parton from the nucleus) probed in direct photon production at forward rapidity in $\mathrm{p}+\mathrm{Pb}$ collisions at $8.8 \mathrm{TeV}$ as calculated in JEPHOX using EPS09 structure functions in Ref. [22]. The different components of photon production are also shown separately. For comparison, the $x_{2}$ distribution for pion production of similar kinematics are included. right: Nuclear modification factor $R_{p A}$ as a function of $p_{T}$ for forward direct photon production. Shown are results of CGC calculations from Ref. [23] (orange) and from NLO pQCD calculations with JETPHOX (blue). The shaded bands show the systematic error estimates. 
[19]. There is also a suppression of $J / \psi$ production [20], which is consistent with calculations using shadowing and final state energy loss. State-of-the-art CGC calculations [21] are also in agreement with the data. In general, also at LHC, hadron production will most likely not provide an ideal probe because of final state modifications and their uncertainties, which will obscure the kinematics.

Probes, which directly access the parton kinematics, would be strongly preferable, which points to direct photons as an ideal probe. Direct photons have a number of advantages compared to other, in particular hadronic probes:

1. The production processes of direct photons are well understood theoretically.

2. The leading order process (q-g Compton scattering) is directly sensitive to the gluon density.

3. Fragmentation contributions, though significant at LHC, are less important than for hadrons, and can be suppressed by isolation cuts.

4. No other strong final state nuclear modification (like e.g. energy loss) is expected.

The advantage of photons with respect to the sensitivity to parton kinematics can be illustrated with the distribution of momentum fractions $x_{2}$ probed in the nucleus in $\mathrm{p}+\mathrm{A}$ reactions at the LHC as displayed in the left panel of Figure 9. The $x_{2}$ distribution for photon production with $4<y<5$ and $5<p_{T}<20 \mathrm{GeV} / c$ is peaked between $10^{-5}$ and $10^{-4}$, while the maximum contribution for pion production is generated from partons with $x_{2}$ about an order of magnitude larger. Thus, already the inclusive direct photon distribution has a clear sensitivity advantage, which can be further enhanced by applying isolation cuts on the photons, although the studies in Ref. [22] show that isolation may not be as effective as previously thought.

Another advantage of photon production is that the theoretical description also in the context of models of gluon saturation is very well understood. State-of-the-art calculations have shown a clear sensitivity to gluon saturation effects, as demonstrated in the right panel of Figure 9, which shows the nuclear modifications factor $R_{p A}$ of direct photons from the CGC calculation in Ref. [23] and from a pQCD calculation at NLO with EPS09 PDFs using JETPHOX. While the pQCD prediction shows only a slight reduction of reaching $R_{p A} \approx 0.8$ at low $p_{T}$ related to nuclear shadowing, the CGC calculation shows a strong suppression to $R_{p A}<0.4$ for $p_{T}<5 \mathrm{GeV} / c$.

In principle the measurement of Drell-Yan production - i.e. virtual direct photons - would provide an alternative means of assessing low-x parton distributions with similar advantages, i.e. no final state modifications. The measurement is not as directly accessing the gluon distribution, however, as gluons play a role only via second order diagrams, or in the quark/antiquark PDFs via DGLAP evolution. It may be an additional complication for the interpretation to rely heavily on DGLAP evolution in theoretical predictions - finally a search for gluon saturation effects should challenge DGLAP evolution. Still this is likely not a major argument against using Drell-Yan.

The major disadvantage of Drell-Yan measurements is the very low cross section compared to real photon production. Ref. [24] shows a measurement of forward Drell-Yan muon pairs in pp collisions at $7 \mathrm{TeV}$ from LHCb. For the low mass range relevant for this discussion $(5<M<$ $7 \mathrm{GeV}$ ) the authors quote a statistical error of $\approx 20 \%$ for the rapidity-integrated measurement in a sample of $37 \mathrm{pb}^{-1}$. A low-mass rapidity-differential measurement would not be possible from this 

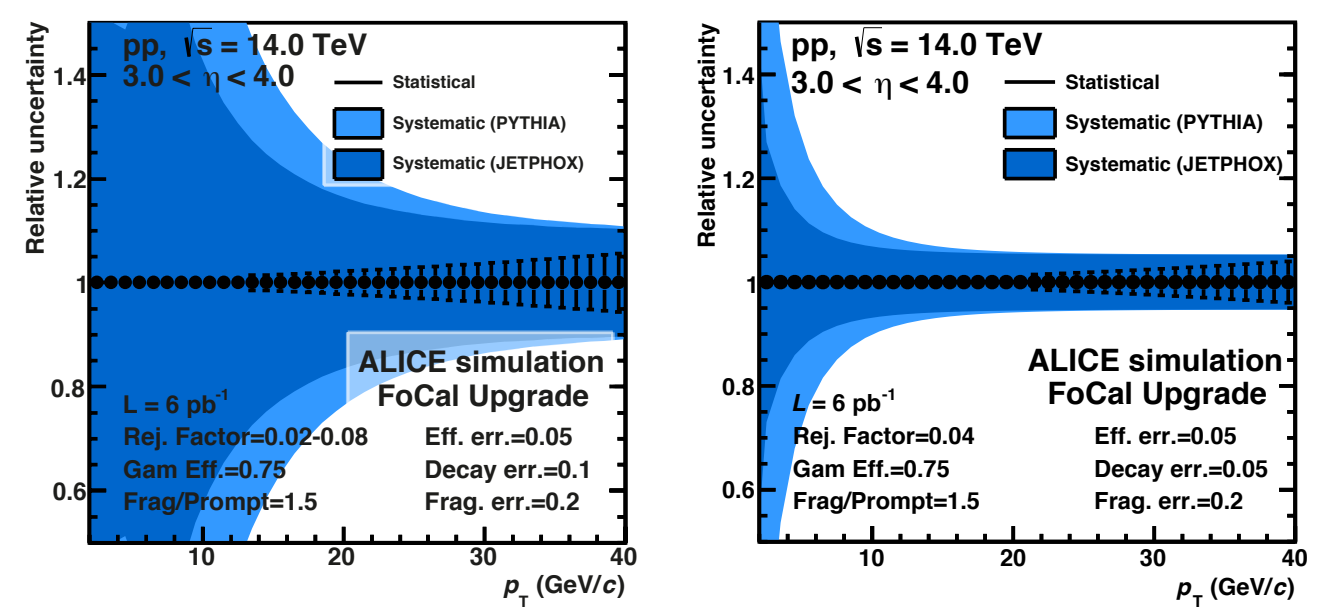

Figure 10: Estimated relative uncertainties on the cross section measurement for direct photon production in $\mathrm{p}+\mathrm{p}$ collisions at $\sqrt{s}=14 \mathrm{TeV}$, based on direct photon spectra from JETPHOX (dark band) and PYTHIA (light band), and background spectra from PYTHIA events. Statistical uncertainties are shown as error bars and the systematic uncertainty is shown as a band. This simulation assumes a location of the detector at $z=3.6 \mathrm{~m}$. Results are shown using only low granularity layers (left) and for the full detector including high granularity layers $(r i g h t)$.

sample. The situation is considerably worse for $\mathrm{p}+\mathrm{Pb}$ collisions, where an integrated luminosity of $50 \mathrm{nb}^{-1}$ (corresponding to a nucleon-nucleon-equivalent luminosity of $\approx 10 \mathrm{pb}^{-1}$ ) is considered reasonable. Measurements of Drell-Yan production will therefore not be competitive to those of real photon production.

Beyond the above upgrade programme, which has been endorsed by the LHC experiments committee (LHCC), an upgrade with a calorimeter at forward rapidities (FoCal) to measure forward direct photon production is currently being discussed in the ALICE collaboration [25]. This detector would be intended to measure direct photons, electrons/positrons and jets for rapidities $\eta>3$. Such a detector would offer a wealth of physics possibilities, but its main focus is on measurements related to the structure of nucleons and nuclei at very low Bjorken- $x$ and possible effects of gluon saturation.

FoCal would consist of an electromagnetic calorimeter most likely positioned at a distance from the IP of $z \approx 7 \mathrm{~m}$ covering $3.2<\eta<5.3$ backed by a standard hadronic calorimeter. A distance of $z=3.6 \mathrm{~m}$, which corresponds to a maximum reachable pseudorapidity of $\eta=4.5$, has also been studied in simulations. Both positions are equivalent in terms of measurement conditions such as the particle density, such that it is sufficient at this stage to not explicitly perform all studies for both positions. The main challenge of an electromagnetic calorimeter in this region of phase space is the requirement to discriminate decay photons from direct photons at very high energy, which will require extremely high granularity.

The baseline design currently under study is a $\mathrm{SiW}$ sandwich construction. It consists of 20 layers of a $3.5 \mathrm{~mm} \mathrm{~W}$ plate $\left(\approx 1 X_{0}\right)$ interleaved with active layers with $\mathrm{Si}$ sensors. The active layers use two different sensor technologies: low granularity layers (LGL), which consist of sensors with $1 \mathrm{~cm}^{2}$ pads summed longitudinally in segments and equipped with analog readout, and high 


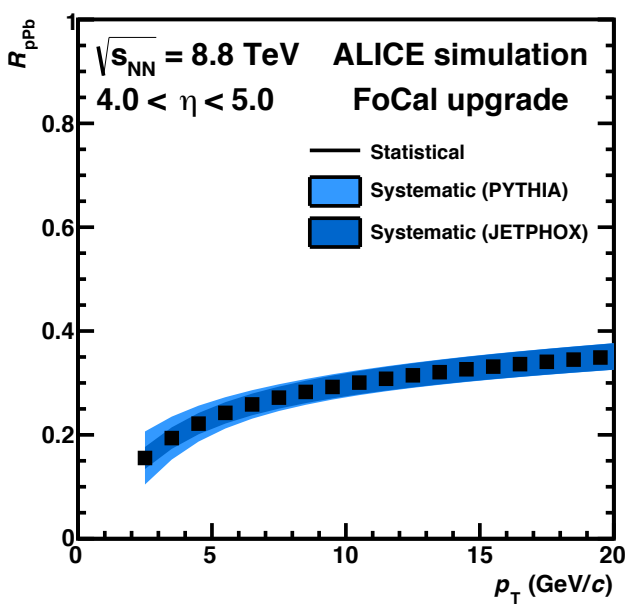

Figure 11: Estimated relative uncertainties on measurement of the nuclear modification factor $R_{p A}$ for direct photon production at $\sqrt{s}=8.8 \mathrm{TeV}$, based on direct photon spectra from JETPHOX (dark band) and PYTHIA (light band), and background spectra from PYTHIA events. Statistical uncertainties are shown as error bars and the systematic uncertainty is shown as a band. This simulation assumes a location of the detector at $z=3.6 \mathrm{~m}$. Results are shown using only low granularity layers (left) and for the full detector including high granularity layers $(r i g h t)$.

granularity layers (HGL) based on CMOS monolithic active pixel sensors (MAPS). The MAPS will have a pixel size of a few $10 \times 10 \mu \mathrm{m}^{2}$ with internal binary readout. ${ }^{1}$ On-chip processing will convert the pixel count in a macro-pixel of $1 \mathrm{~mm}^{2}$ to a pseudo-analog value.

The HGL are crucial for $\gamma / \pi^{0}$ discrimination. The LGL have an effective tower width of the order of the Molière radius. Their two-shower separation power is similar to existing standard electromagnetic calorimeters. ${ }^{2}$ However, the shape of electromagnetic showers allows us to make use of much finer granularity for shower separation and additional shower shape analysis for very high energy $\pi^{0}$, when the two photons can no longer be resolved. The impact of the granularity is shown in Fig. 10, which displays uncertainty estimates for a direct photon measurement in pp collisions at $14 \mathrm{TeV}$. The panel on the left hand side shows the expected performance using only the LGL, while the right panel shows the performance of the full detector. A low-granularity detector would only determine the photon yield with a much larger systematic error, mainly due to the merging of $\pi^{0}$-decay photons. Only the high-granularity option has a good sensitivity for such a photon measurement. While FoCal would offer coverage towards higher rapidities than other LHC experiments, it is in particular the superior granularity at these large rapidities that would give FoCal a unique advantage. The estimated performance on the flagship measurement of the nuclear modification factor $R_{p A}$ is shown in Fig. 11.

The detector upgrade would be installed in LS $3(\approx 2024)$, and measurements would be performed together with the full ALICE setup. Beam intensity conditions should thus be similar to the standard requirements of the upgraded ALICE experiment.

\footnotetext{
${ }^{1}$ The current model for MC simulations uses a pixel size of $100 \times 100 \mu \mathrm{m}^{2}$.

${ }^{2}$ Those conditions are in fact very similar to the ones of the electromagnetic calorimeter of the LHCb experiment.
} 


\section{The future: FCC}

For the still longer term future the opportunities for heavy-ion physics are investigated in the context of a design study for the Future Circular Collider (FCC) [26]. Such a facility could provide beam energies of $\sqrt{s}=100 \mathrm{TeV}$ for pp collisions, and correspondingly $\sqrt{s_{N N}}=63 \mathrm{TeV}$ for $\mathrm{p}-\mathrm{Pb}$ and $39 \mathrm{TeV}$ for $\mathrm{Pb}-\mathrm{Pb}$ collisions. From simple estimates this should correspond to multiplicity densities and volumes about $2 \times$ larger than at LHC with a life time $\approx 20 \%$ larger. This should also result in significantly higher energy density and temperature. In particular one expects a sizeable secondary production of charm and anti-charm quarks pairs, which should be very sensitive to the initial temperature and system evolution.

But the higher beam energy and the higher expected luminosity will also make new rare hard probes accessible. As an example, it is estimated that the cross section for top quark production increases by a factor 80 compared to LHC, so that a high statistics sample of top quark pairs can be obtained, which would offer unique new opportunities.

Furthermore the higher beam energy very naturally allows to access much lower Bjorken $x$ values than previously possible (down to $x \approx 10^{-7}$ ) both in hadronic interactions (in particular $\mathrm{p}$ $\mathrm{Pb}$ ) and in ultra-peripheral collisions, giving unique information about the gluon density at low $x$ and possible saturation effects. Finally, measurements at FCC would make a crucial step in closing the gap between accelerator measurements and cosmic rays at the highest energies and will thus help in understanding cosmic ray showers in the atmosphere.

\section{Summary}

Heavy-ion physics still has a rich future ahead at LHC and beyond. Rare, triggered probes, like high $E_{T}$ jets or weak bosons, will profit from the higher luminosity at LHC. This is in particular the realm of ATLAS and CMS. The upgrade programmes of those experiment will thus also be of benefit for heavy-ion studies, and we are awaiting much more significant data sets on these rare probes.

ALICE with its different philosophy concentrating on rare, but untriggerable probes will contribute strongly in the future, in particular on charm measurements and low $p_{T}$ (and mass) photons and di-leptons, and on all observables profiting from particle identification. This programme will only be possible with a dedicated upgrade of the core ALICE detectors. An extensive R\&D programme has been launched to meet the challenges of high-rate running with improved performance. A particular additional opportunity exists for gluon saturation studies via the measurement of forward direct photons. For this a new forward calorimeter (FoCal) would be needed, which is currently under discussion.

Finally, opportunities may arise for heavy-ion physics at still higher beam energy at a possible new accelerator, the FCC. Studies of the theoretical possibilities and the detector requirements have just been started.

\section{Acknowledgements}

The author would like to thank B. Wyslouch, P. Steinberg, and M. van Leeuwen for providing important information. 


\section{References}

[1] B. Abelev et al. (ALICE Collaboration), Phys. Rev. Lett. 109 (2012) 252301; B. Abelev et al. (ALICE Collaboration) Phys. Rev. C 88 (2013) 044910.

[2] M. Floris et al. (ALICE Collaboration), J.Phys.G 38 (2011)124025.

[3] K. Aamodt et al. (ALICE Collaboration), Phys. Rev. Lett. 107 (2011) 032301; B. Abelev et al. (ALICE Collaboration), JHEP 06 (2015) 190; G. Aad et al. (ATLAS Collaboration), Phys. Lett. B707 (2012) 330; S. Chatrchyan et al. (CMS Collaboration), Eur. Phys. C 72 (2012) 10052.

[4] B. Abelev et al. (ALICE Collaboration), Phys. Rev. Lett. 109 (2012) 072301.

[5] P. Braun-Munzinger and J. Stachel, (2009), arXiv:0901.2500.

[6] K. Aamodt et al. (ALICE Collaboration), Phys. Lett. B696 (2011) 30; S. Chatrchyan et al. (CMS Collaboration), Eur. Phys. J. C 72 (2012) 1945; A. Milov et al. (ATLAS Collaboration), J.Phys.G G38 (2011) 124113.

[7] G. Aad et al. (ATLAS Collaboration), Phys. Rev. Lett. 105 (2010) 252303; S. Chatrchyan et al. (CMS Collaboration), Phys. Rev. C 84 (2011) 024906.

[8] B. Abelev et al. (ALICE Collaboration), JHEP 09 (2012) 112.

[9] B. Abelev et al. (ALICE Collaboration), Phys. Rev. C 90 (2014) 034904.

[10] ATLAS Collaboration, CERN-LHCC-2011-012; CERN-LHCC-2012-022.

[11] CMS Collaboration, CERN-LHCC-2011-006; CERN-LHCC-2015-010.

[12] ALICE Collaboration, CERN-LHCC-2012-012.

[13] K. Aamodt et al. (ALICE), JINST 3, S08002 (2008).

[14] ALICE Collaboration, CERN-LHCC-2012-013; CERN-LHCC-2013-024.

[15] ALICE Collaboration, CERN-LHCC-2013-014.

[16] L.V. Gribov, E.M. Levin, and M.G. Ryskin, Phys. Rept. 100 (1983) 1Đ150; A.H. Mueller and J.-W. Qiu, Nucl. Phys. B268 (1986) 427; L.D. McLerran and R. Venugopalan, Phys. Rev. D49 (1994) 223-2241; L.D. McLerran and R. Venugopalan, Phys. Rev. D49 (1994) 335-3355; L.D. McLerran and R. Venugopalan, Phys. Rev. D50 (1994) 222-2233.

[17] J. Adams et al. (STAR Collaboration), Phys. Rev. Lett. 97 (2006) 152302; I. Arsene et al. (BRAHMS Collaboration) Phys. Rev. Lett. 93 (2004) 242303.

[18] E. Braidot et al. (STAR collaboration), Proceedings of the 45th Rencontres de Moriond (2010) preprint arXiv:1005.2378; A. Adare et al. (PHENIX Collaboration), Phys. Rev. Lett. 107, 172301 (2011).

[19] A. Uras, ALICE Collaboration, talk at Hard Probes 2013.

[20] LHCb Collaboration, JHEP 02 (2014) 072; B. Abelev et al. (ALICE Collaboration), JHEP02 (2014) 073.

[21] Y.-Q. Ma, R. Venugopalan, and H.-F. Zhang, arXiv:1503.07772.

[22] I. Helenius, K.J. Eskola, H. Paukkunen, preprint arXiv:1406.1689.

[23] J. Jalilian-Marian, A. Rezaeian, Phys. Rev. D 86 (2012) 034016; A. Rezaeian, private communication. 
[24] LHCb collaboration, LHCb-CONF-2012-013.

[25] T. Peitzmann, Proceedings of CHEF 2013, preprint arXiv:1308.2585.

[26] N. Armesto et al., Nucl. Phys. A 931 (2014) 1163-1168. 